\title{
Development and implementation of high-throughput SNP genotyping in barley
}

Timothy J Close*1, Prasanna R Bhat ${ }^{1,12}$, Stefano Lonardi ${ }^{2}$, Yonghui $\mathrm{Wu}^{2,13}$, Nils Rostoks ${ }^{3,14}$, Luke Ramsay ${ }^{3}$, Arnis Druka ${ }^{3}$, Nils Stein ${ }^{4}$, Jan T Svensson ${ }^{1,15}$, Steve Wanamaker ${ }^{1}$, Serdar Bozdag2,16, Mikeal L Roose ${ }^{1}$, Matthew J Moscou ${ }^{1,17}$, Shiaoman Chao ${ }^{5}$, Rajeev K Varshney ${ }^{4,18}$, Péter Szűcs ${ }^{6}$, Kazuhiro Sato ${ }^{7}$, Patrick M Hayes ${ }^{6}$, David E Matthews ${ }^{8}$, Andris Kleinhofs 9 , Gary J Muehlbauer ${ }^{10}$, Joseph DeYoung ${ }^{11}$, David F Marshall ${ }^{3}$, Kavitha Madishetty ${ }^{1}$, Raymond D Fenton ${ }^{1}$, Pascal Condamine ${ }^{1,19}$, Andreas Graner ${ }^{4}$ and Robbie Waugh ${ }^{3}$

Address: ${ }^{1}$ Department of Botany \& Plant Sciences, University of California (UCR), Riverside, CA, 92521, USA, ${ }^{2}$ Department of Computer Sciences, University of California (UCR), Riverside, CA, 92521, USA, ${ }^{3}$ Scottish Crop Research Institute (SCRI), Invergowrie, Dundee, DD2 5DA, UK, ${ }^{4}$ Leibniz Institute of Plant Genetics and Crop Plant Research (IPK), Corrensstrasse 3, D-06466, Gatersleben, Germany, ${ }^{5}$ USDA-ARS Biosciences Research Lab, Fargo, ND, 58105-5674, USA, ${ }^{6}$ Department of Crop and Soil Science, Oregon State University, Corvallis, OR, 97331, USA, ${ }^{7}$ Research Institute for Bioresources, Okayama University, Kurashiki, 710-0046, Japan, ${ }^{8}$ USDA-ARS, Cornell University, Ithaca, NY, 14853, USA, ${ }^{9}$ Department of Crop and Soil Sciences, Washington State University, Pullman, WA, 99164, USA, ${ }^{10}$ Department of Agronomy and Plant Genetics, University of Minnesota, St. Paul, MN, 55108, USA, ${ }^{11}$ Southern California Genotyping Consortium, University of California, Los Angeles, CA, 90095, USA, ${ }^{12}$ Monsanto Research Centre, Bangalore, 560092, India, ${ }^{13}$ Google, Mountain View, CA, 94043, USA, ${ }^{14}$ Faculty of Biology, University of Latvia, 4 Kronvalda Boulevard, Riga, LV-1586, Latvia, ${ }^{15}$ University of Copenhagen, Frederiksberg C, DK-1871, Denmark, ${ }^{16}$ NIH National Cancer Institute, Neuro-Oncology Branch, Bethesda, MD, 20892, USA, ${ }^{17}$ Department of Plant Pathology, Iowa State University, Ames, Iowa, 50011, USA, ${ }^{18}$ International Crops Research Institute for the Semi-Arid Tropics (ICRISAT), Patancheru - 502 324, Andhra Pradesh, India and ${ }^{19}$ NetSocial Marketing, Le Puech, 15600 Montmurat, France

Email: Timothy J Close* - timothy.close@ucr.edu; Prasanna R Bhat - prasannarb@gmail.com; Stefano Lonardi - stelo@cs.ucr.edu; YonghuiWu -yonghui@cs.ucr.edu; Nils Rostoks - nrostoks@latnet.lv; Luke Ramsay - Luke.Ramsay@scri.ac.uk; Arnis Druka - arnisdruka@aol.com; Nils Stein - stein@ipk-gatersleben.de; Jan T Svensson - jsv@life.ku.dk; Steve Wanamaker - steve.wanamaker@ucr.edu; Serdar Bozdag - sbozdag@cs.ucr.edu; Mikeal L Roose - mikeal.roose@ucr.edu; Matthew J Moscou - moscou@iastate.edu; Shiaoman Chao - Shiaoman.Chao@ars.usda.gov; Rajeev K Varshney - r.k.varshney@cgiar.org; Péter Szücs - Peter.Szucs@oregonstate.edu; Kazuhiro Sato - kazsato@rib.okayama-U.ac.jp; Patrick M Hayes - hayesp@css.orst.edu; David E Matthews - Matthews@greengenes.cit.cornell.edu; Andris Kleinhofs - andyk@wsu.edu; Gary J Muehlbauer - Gary.J.Muehlbauer$1 @$ tc.umn.edu; Joseph DeYoung - Jdeyoung@mednet.ucla.edu; David F Marshall - D.F.Marshall@scri.sari.ac.uk;

Kavitha Madishetty - kavithavcs@yahoo.com; Raymond D Fenton - raymond.fenton@ucr.edu; Pascal Condamine - pascalcondamine@yahoo.fr; Andreas Graner - a_graner@IPK-Gatersleben.de; Robbie Waugh - rwaugh@scri.sari.ac.uk

* Corresponding author 
in consensus maps and problematic when fine resolution is critical including comparative genome analyses and map-based cloning. Here we provide a new paradigm, a high-density consensus genetic map of barley based only on complete and error-free datasets and genic markers, represented accurately by graphs and approximately by a best-fit linear order, and supported by a readily available SNP genotyping resource.

Results: Approximately 22,000 SNPs were identified from barley ESTs and sequenced amplicons; 4,596 of them were tested for performance in three pilot phase Illumina GoldenGate assays. Data from three barley doubled haploid mapping populations supported the production of an initial consensus map. Over 200 germplasm selections, principally European and US breeding material, were used to estimate minor allele frequency (MAF) for each SNP. We selected 3,072 of these tested SNPs based on technical performance, map location, MAF and biological interest to fill two I536-SNP "production" assays (BOPAI and BOPA2), which were made available to the barley genetics community. Data were added using BOPAI from a fourth mapping population to yield a consensus map containing 2,943 SNP loci in 975 marker bins covering a genetic distance of 1099 cM.

Conclusion: The unprecedented density of genic markers and marker bins enabled a high resolution comparison of the genomes of barley and rice. Low recombination in pericentric regions is evident from bins containing many more than the average number of markers, meaning that a large number of genes are recombinationally locked into the genetic centromeric regions of several barley chromosomes. Examination of US breeding germplasm illustrated the usefulness of BOPAI and BOPA2 in that they provide excellent marker density and sensitivity for detection of minor alleles in this genetically narrow material.

\section{Background}

Complete genome sequences of many plants, including economically important small grain cereals such as barley, are unlikely to be available in the near future if they have large genomes and contain much repetitive DNA. The barley genome is $5200 \mathrm{Mbp}$, which is more than twelve times rice, and composed of at least $80 \%$ highly repetitive DNA, which is likely to preclude a whole-genome assembly from shotgun sequences obtained with currently available technologies. However, access to most of the genes of barley and numerous other organisms can be gained through cDNAs (generally expressed sequence tags; ESTs) and sequenced PCR amplicons, which provide a facile route to single nucleotide polymorphisms (SNPs) in proteinencoding transcribed genes. As of the January 2, 2009 release of dbEST, there were 525,527 Sanger-sequenced ESTs from barley. These were derived principally from eight malting barley cultivars and one wild barley accession, with a minor fraction from several other barley genotypes. Here we describe the use of the majority of this transcriptome sequence resource to develop highthroughput SNP genotyping in barley, application of the new SNP methods to the production of a high-density and high quality SNP map that can be related readily to prior maps through shared markers and other grass genomes through synteny, and deployment of these new resources in support of marker-assisted breeding and association genetic analyses.
In recent years there has been a surge in marker density and convergence toward consensus maps for barley. Rostoks et al. [1] developed a consensus map containing 1230 markers (RFLP, AFLP, SSR, SNP) from three doubled haploid populations. Wenzl et al. [2] combined DArT with RFLP, SSR and STS from nine mapping populations to create a consensus map containing 2935 markers. Marcel et al. [3] compiled RFLP, AFLP and SSR data from six mapping populations to produce a consensus map containing 3458 markers. Stein et al. [4] used three doubled haploid mapping populations and combined new data from 1,055 markers (RFLP, SSR, SNP) with prior data from 200 anchor markers to produce a 1255 marker consensus map. Varshney et al. [5] produced a 775 SSR consensus map by joining six independent maps. Potokina et al. [6] combined SNP and other transcript derived markers to position 1596 loci on the Steptoe $\times$ Morex [7] linkage map. Hearnden et al. [8] combined 1000 SSR and DArT markers on a map from a wide cross. Several additional maps which have used portions of the SNP data described in the present work have been published or are nearing publication including a 2890 SNP and STS map from the Haruno Nijo $\times$ OUH602 population [9] and a 2383 marker map (DArT, SNP, SSR, AFLP, RFLP, STS, QTL) from the Oregon Wolfe Barley population [10], among others. Marker intersection between these maps is significant, but missing data, non-uniform data quality and anonymity of many markers constrain the accuracy of 
the map merging process and the resolution of synteny between barley and other genomes. Here we describe a new element of the map convergence equation, a high fidelity and dense consensus map produced entirely from transcribed gene SNPs using only a very robust portion of genotyping data derived from four mapping populations utilizing the Illumina GoldenGate assay (Illumina Inc., San Diego, CA). Maps that include SNPs in protein-coding genes facilitate genome content comparisons by virtue of the high conservation of protein sequences across genera, thus enabling sequence similarity searches to find orthologs. The SNPs and data described herein have been made available incrementally in parallel with their production since mid-2005 to the barley community to facilitate research. Here we provide full details of the development of the SNP genotyping platform and some of the insight it has brought.

\section{Results and Discussion Identification of SNPs and development of Golden Gate Assays}

Details of the identification of approximately 22,000 SNPs from EST and PCR amplicon sequence alignments, and development of three test phase and two production scale Illumina GoldenGate oligonucleotide pool assays (OPAs), are briefly summarized in Methods and provided more fully in Supplemental Text (Additional File 1). In total, 4596 SNPs were tested using 576 DNA samples on pilot OPAs POPA1 and POPA2, and 480 DNA samples on POPA3, followed by selection of 3072 technically satisfactory and genetically most informative SNPs for representation on two production OPAs (BOPA1, BOPA2) (Figure 1). Of these 4596 SNPs, 3456 originated from ESTs and 1140 from PCR amplicons derived from genomic sequences. Of the 3072 SNPs selected for two production OPAs, 2279 were from ESTs and 793 from PCR amplicons. There was considerable intersection in the sets of SNPs provided by each identification path. For all OPAs preference was given to SNPs identified by amplicon sequencing. The final tally of surviving SNPs from each selection path included $65.9 \%(2279 / 3456)$ of the ESTderived and $69.6 \%(793 / 1140)$ of the PCR ampliconderived SNPs. By this metric, the overall success rates were essentially equal for the two strategies for SNP discovery, ESTs versus genomic amplicon sequences.

The yield of SNPs from each of 253 pairwise genotype alignments of ESTs (see Supplemental Text for SNP selection details, Additional File 1) revealed a strong linear relationship $\left(\mathrm{r}^{2}=0.84\right)$ between the number of SNPs and the product of the number of ESTs. For example, the initial set of 36 pairwise genotype comparisons between eight malting barley cultivars and one wild barley accession (used for POPA1 and POPA2) is provided in Figure S1, Additional File 2), where this linear relationship and

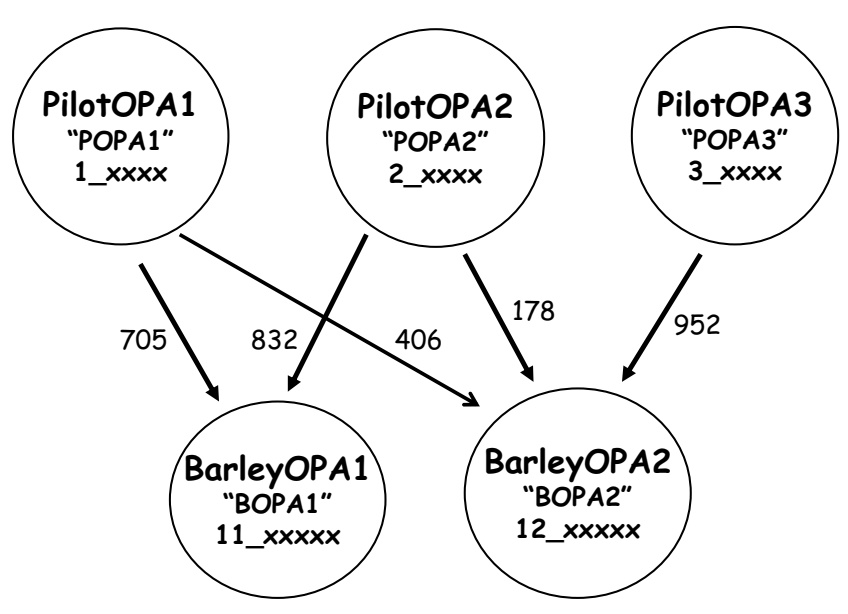

Figure I

Five I 536-plex GoldenGate assays. The numbers of SNPs selected from each Pilot OPA (POPAI, POPA2, POPA3) for the design of each production scale OPA (BOPAI, BOPA2) are indicted next to the arrows connecting the pilot and production OPAs. See Supplemental Text (Additional File I) for complete details.

the higher frequency of SNPs when including the wild barley accession are readily apparent. In retrospect, it was fortuitous for SNP discovery that researchers in each country chose their own local favourite malting barley for EST sequencing.

\section{Genetic linkage maps}

For each of the four mapping populations the linkage groups separated cleanly using MSTMap (see Methods) at LOD 4 or 5 and generally remained intact at higher LOD values. The four maps from individual crosses were fused using MergeMap (see Methods) to form a consensus map containing 2943 SNP loci with a total map length of 1099 $\mathrm{cM}$ (Table 1). The identity and polarity of linkage groups were determined by integrating 110 previously mapped bin markers [11] into the SxM and consensus maps (Table S1, Additional File 3). Because the SNP data are more complete and seem generally to be of higher quality than the SxM bin marker data, the 2943 "SNP-only" map and its distance coordinates are taken as the central point of reference in this paper (Figure S2, Additional File 4). Table S1 (Additional File 3) provides map coordinates for each of the four individual maps, the SxM map with 110 bin markers, the 2943 SNP-only consensus map and the 3053 marker consensus map containing 2943 SNPs and 110 SxM bin markers. The number and distribution of loci for each individual SNP-only map and the consensus SNPonly map are given in Table 1 . In all maps, chromosome $5 \mathrm{H}$ has the greatest length, a mean of $198 \mathrm{cM}$, consistent with previously published linkage maps. Chromosome $5 \mathrm{H}$ is also the most populated with $535 \mathrm{SNP}$ loci and is 
Table I: Distribution of SNPs in four individual maps and consensus map

\begin{tabular}{|c|c|c|c|c|c|c|c|c|c|}
\hline \multirow[b]{2}{*}{ Map } & \multirow[b]{2}{*}{ Count type } & \multicolumn{8}{|c|}{ Chromosome } \\
\hline & & $\mathbf{I H}$ & $2 \mathrm{H}$ & $3 \mathbf{H}$ & $4 \mathrm{H}$ & $5 \mathbf{H}$ & $6 \mathrm{H}$ & 7H & All \\
\hline \multirow[t]{3}{*}{ Morex $\times$ Barke } & markers & 215 & 279 & 246 & 141 & 299 & 219 & 248 & 1652 \\
\hline & bins & 60 & 72 & 77 & 39 & 74 & 54 & 65 & 443 \\
\hline & $\mathbf{c M}$ & 134.0 & 151.9 & 178.1 & 112.4 & 195.7 & 133.8 & 158.9 & 1064.9 \\
\hline \multirow[t]{3}{*}{ Oregon Wolfe Barley } & markers & 168 & 235 & 255 & 211 & 278 & 202 & 213 & 1562 \\
\hline & bins & 65 & 73 & 91 & 60 & 89 & 64 & 67 & 509 \\
\hline & cM & 145.4 & 181.0 & 199.3 & 121.8 & 231.1 & 152.3 & 186.7 & 1217.6 \\
\hline \multirow[t]{3}{*}{ Steptoe $\times$ Morex } & markers & 148 & 217 & 242 & 130 & 225 & 122 & 183 & 1270 \\
\hline & bins & 49 & 57 & 63 & 49 & 80 & 40 & 57 & 396 \\
\hline & $\mathbf{c M}$ & 139.7 & 148.8 & 154.7 & $|4| .5$ & 187.3 & 123.8 & 140.8 & 1036.6 \\
\hline \multirow[t]{3}{*}{ Haruna Nijo × OHU602 } & markers & 93 & 131 & 123 & 97 & 108 & 92 & 88 & 732 \\
\hline & bins & 46 & 65 & 58 & 48 & 58 & 40 & 47 & 362 \\
\hline & $\mathbf{c M}$ & 145.2 & 162.6 & 162.7 & 124.5 & 176.4 & 123.0 & 182.5 & 1076.7 \\
\hline \multirow[t]{3}{*}{ Consensus } & markers & 341 & 485 & 475 & 338 & 535 & 352 & 417 & 2943 \\
\hline & bins & 125 & 161 & 152 & 113 & 180 & 111 & 133 & 975 \\
\hline & $\mathbf{c M}$ & $14||$. & 161.1 & 173.7 & 125.1 & 197.6 & 133.2 & 167.2 & 1099.0 \\
\hline
\end{tabular}

subdivided into the largest number of marker bins (180). On the lower end of the spectrum chromosome $4 \mathrm{H}$ has only 338 SNP loci distributed among 113 marker bins covering $125 \mathrm{cM}$. The relationship of nearly one marker bin per $\mathrm{cM}$ holds for all seven linkage groups.

Once the SNP loci were arranged by position on the consensus map, graphical visualization enabled inspection of the distribution of recombination events. The genotype data and graphical genotype displays for three of the four mapping populations $(\mathrm{MxB}, \mathrm{OWB}, \mathrm{SxM})$ are provided in Table S2 (Additional File 5), where it can be seen that there are no singleton double recombinant loci in densely marked regions of any of the maps. Since such loci are often indicative of genotyping errors, the complete absence of suspicious double recombinants can be considered an indicator of high fidelity of the data from the 2943 SNP loci selected for linkage map production. Other quality metrics include the frequency of missing data or apparent heterozygosity; aside from two instances of apparent heterozygosity at locus 1_1166 in two seemingly identical OWB doubled haploid lines \#22 and \#70 (Figure 2D, Table S2, Additional File 5), all individuals in all three mapping populations had homozygous genotype calls for all loci and no missing data. This is $100 \%$ of 153,636 possible genotype calls in the $\mathrm{MxB}$ population, $99.999 \%$ of 145,266 possible genotype calls in the OWB population and $100 \%$ of 116,840 possible genotype calls in the SxM population. The high fidelity and lack of missing data among these selected 2943 SNPs facilitated the production of individual and consensus maps. More than 300 SNPs with imperfect but still high quality data (for example 3_1104, Figure 2C) were not utilized for this map.

Figure 3 illustrates the number of shared markers between any two, any three and all four maps. The substantial number of shared markers facilitated the production of a consensus map. The number of pairwise shared markers ranged from 303 between the $\mathrm{HxO}$ and OWB maps to 786 shared between the MxB and SxM maps. Three-way shared 


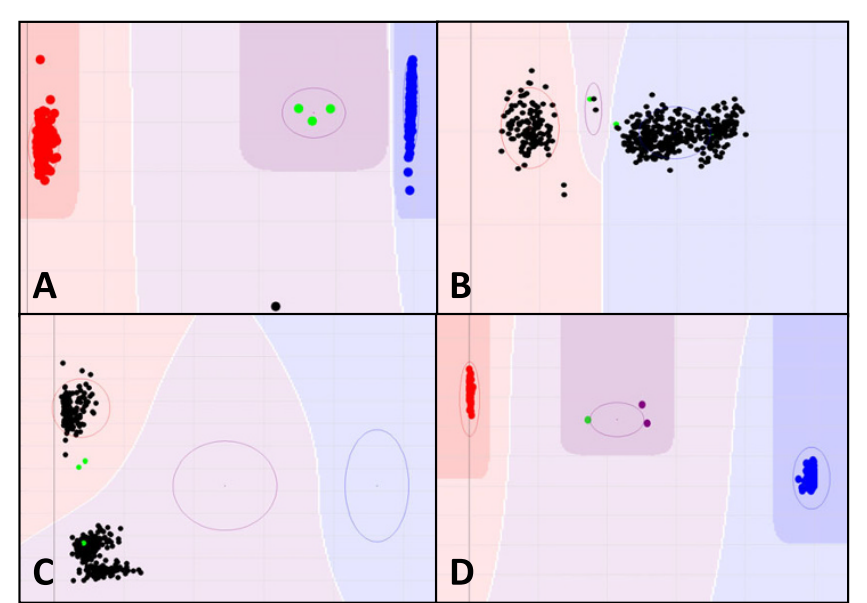

Figure 2

Examples of SNP data. A) Typical clustering of satisfactory data for POPA SNP 3_0004; red cluster area = homozygous $A A$, blue $=$ homozygous $B B$, green dots within purple cluster area are I:I mixtures of parental DNA for three DH mapping populations. One germplasm sample (black dot) was outside of any call cluster and was thus scored "no call". B) Typical theta compressed data for POPA SNP 3_I I04; although the polymorphism can be mapped in an individual population there are often wrong calls in such data and the cluster separation is problematic for general use in germplasm analyses or with multiple mapping populations; set to Gentrain 0.000 , 100\% "no call". C) Typical vertically separated clusters for POPA SNP 3_0070; generally polymorphic for a different locus than the source of the targeted SNP, which results in wrong annotation and degraded synteny; set to Gentrain 0.000, I00\% "no call". D) Data for POPA SNP I_I I 66 (ABC07305-I-4-322) from the OWB population; two $\mathrm{DH}$ samples behave as heterozygotes (purple cluster), far from the homozygotes (red = AA; blue = $\mathrm{BB}$ ), instead with the I:I mixture of parental DNAs (green dot in purple cluster).

markers range from 120 when including all maps except $\mathrm{MxB}$ to 321 when including all maps except $\mathrm{HxO}$. The lower number of shared markers involving the HxO map is due to the fact that this population was genotyped using only BOPA1, whereas the other three populations were genotyped using all three Pilot OPAs (see Methods). Table S1 (Additional File 3) provides complete information on the map locations of all markers, where it can also be seen that there was no disagreement in the order of shared markers in any of the six pairwise comparisons of linkage maps, or between the consensus map and any individual map. It should be noted, however, that this does not guarantee that the marker order in the 2943-SNP consensus map perfectly matches the order of the corresponding nucleotides within the genome sequence. The consensus map is simply one of many possible non-conflicting linear representations of the consensus DAGs (Figure 4, Figures S3-S9, Additional Files 6, 7, 8, 9, 10, 11 and 12). The limit of knowledge of non-shared marker order is more accurately shown in the consensus DAGs of each linkage group. As more data accumulate from additional mapping populations, linkage disequilibrium analyses and genome sequencing, the number of non-conflicting linear map orders will be reduced, ideally to just one possible order. Naturally, the consensus map will evolve toward finer resolution and convergence on the correct order of all markers.

Segregation distortion was observed in all four mapping populations, being most pronounced in the $\mathrm{MxB}$ population in the pericentric regions of $1 \mathrm{H}, 2 \mathrm{H}, 5 \mathrm{H}$ and $7 \mathrm{H}$ and the long arm of $7 \mathrm{H}$. Interestingly, on $7 \mathrm{H}$ the distortion was toward the maternal allele (Morex) in the pericentric region but toward the paternal allele (Barke) on the long arm.

\section{Alternative marker names}

Table S3 (Additional File 13) provides a cross-reference between synonymous marker names, relating SNPs mapped in the present work to the same genes mapped previously using other marker systems [3]. To generate this cross reference, all of the HarvEST:Barley assembly \#35 unigenes (U35; Table S4, Additional File 14) were searched using BLASTN against the GrainGenes "Sequenced Probes" database http://wheat.pw.usda.gov/ GG2/index.shtml at a cut-off of 1e-10. Probes that matched more than three U35 unigenes were ignored. The result was 636 previously mapped probes matching 1114 unigenes. The list of probes was then reduced to those mapped in Marcel et al. [3] and the list of unigenes was limited to those which were sources of the 2943 mapped SNPs. Finally, the map position of the SNP and the previously mapped probe were compared, discarding a few with gross mismatches in genome location (presumably paralogous loci mapped by the probe). The resulting intersection contains 55 SNPs representing 51 U35 unigenes matching 51 sequenced probes. By comparing the map positions in Table S3 (Additional File 13) one can see that there is perfect co linearity of shared marker order between the two maps, though there are differences in map distances throughout each linkage group. There are no shared markers on $4 \mathrm{HL}$, which illustrates the need for a more comprehensive cross-reference resource than just these two consensus maps. A similar operation can be performed to relate other maps to the present 2943 SNP map. It should be noted also that the original SNP names from SCRI ("ABC" format, Table S4, Additional File 14) correspond in many cases to SNPs used in Rostoks et al. [1] and the original SNP names from IPK ("ConsensusGBS" format, Table S4, Additional File 14) correspond to SNPs in Kota et al. [12]. Thus, those two maps can be readily crossreferenced to the present map using in-common marker names. Also, as stated above, 110 bin markers from the 


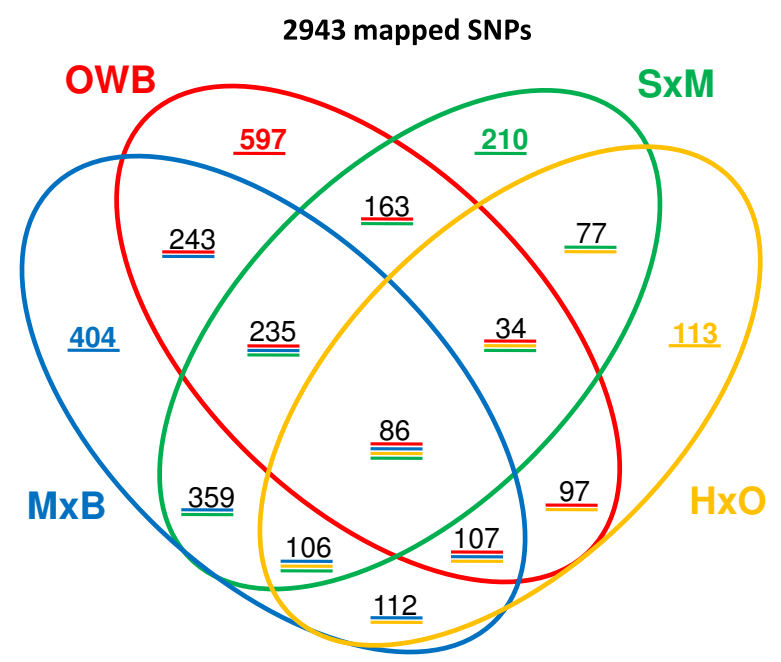

Figure 3

Venn diagram showing marker overlap. A four-way Venn diagram illustrates all unique, two-way, three-way and four-way sets of shared markers. The mapping populations are abbreviated as in the text: $M \times B=$ Morex $\times$ Barke, OWB = Oregon Wolfe Barley, SxM = Steptoe $\times$ Morex, $\mathrm{HxO}=$ Haruna Nijo $\times$ OHU602.

SxM map of Kleinhofs and Graner [11] are included in Table S1 (Additional File 3). Overall, cross-referencing the 2943 SNP-only map to previous maps provides an important bridge between additional resources including a physical map now being coupled to the 2943 SNP-only map and QTLs, simple trait determinants and deletion sites that already have been mapped in prior work. Szücs et al. [10] included 1472 of the SNPs developed in the present work in addition to SSRs, AFLPs and DArT markers, making the resulting OWB map an excellent new point of cross-reference for barley markers.

\section{Synteny}

Each barley SNP source sequence was compared to the rice (Oryza sativa) version 5 and version 6 gene models [13] using BLASTX, and the top hit was taken as the most similar rice gene. These rice best hit coordinates were used as the basis of alignments of each of the seven barley chromosomes with the twelve rice chromosomes. Figure 5 is a screen shot from HarvEST:Barley [14] showing a detailed alignment of barley chromosome $5 \mathrm{H}$ with rice chromosomes. From this and each of the other six barley-rice alignments the marker density is sufficient to clearly reveal major elements of barley-rice synteny, consistent in general with prior publications on Triticeae-rice synteny (for example $[15,16]$. The short arm of barley $5 \mathrm{H}$ is syntenic with rice $12 \mathrm{~L}$. The long arm of barley $5 \mathrm{H}$ is syntenic with an interspersion of rice $12 \mathrm{~S}$ and $11 \mathrm{~S}$ genes followed by rice $9 \mathrm{~S}$, then rice $9 \mathrm{~L}$, then rice $3 \mathrm{~L}$. The position of the centromere in each barley chromosome was determined using flow-sorted chromosome arms in work that will be described in detail elsewhere (Prasanna Bhat et al. in preparation). Of the seven barley chromosomes, $5 \mathrm{H}$ has the most complex barley-rice synteny relationship, being the only barley chromosome composed of major syntenous blocks from more than two ancestors of rice chromosomes. An illustration of barley-rice synteny for all seven barley chromosomes is provided in Figure 6. The simplest relationships are essentially total synteny between barley $3 \mathrm{H}$ versus rice $1(3 \mathrm{HS}=1 \mathrm{~S}, 3 \mathrm{HL}=1 \mathrm{~L})$ and barley $6 \mathrm{H}$ versus rice $2(6 \mathrm{HS}=2 \mathrm{~S}, 6 \mathrm{HL}=2 \mathrm{~L})$. The four remaining barley chromosomes each are composed of ancestors of two rice chromosomes, in each case having one ancestral chromosome nested within the pericentric region, flanked by segments of the other syntenic chromosome. Detailed views of synteny similar to Figure 5, but with zoom-in and active links to external databases, are available for all seven chromosomes through the Windows version of HarvEST:Barley [14].

An interesting consequence of the evolutionary history of barley chromosomes is that the number of expressed genes in the pericentric regions is highly variable, ranging from relatively few in the cases of barley $3 \mathrm{H}$ and $6 \mathrm{H}$ to very many in the most extreme case of barley $7 \mathrm{H}$. The relative genetic map density of expressed genes has major implications for plant breeding efforts. If, for example, a trait maps to an ancestral telomeric region within the pericentric region of barley $7 \mathrm{H}$ then it will be much less likely that the gene controlling that trait can be separated from neighbouring genes by recombination than, for example, a gene located in the ancestral centromeric region of rice chromosome 9, which is located in a more recombinationally active region on barley chromosome $5 \mathrm{H}$. For example there have been several as yet unsuccessful attempts to map-base clone $\mathrm{Mlg}$, a powdery mildew resistance gene located in the gene-dense pericentric region of 4H (Matthew Moscou, unpublished data). Similarly, the level of difficulty in map-based cloning efforts will also depend on the gene density in regions of low recombination. Due to high gene density in ancestral telomeric regions which are now nested within barley chromosomes, the pericentric regions of five barley chromosomes $(1 \mathrm{H}, 2 \mathrm{H}, 4 \mathrm{H}, 5 \mathrm{H}$ and $7 \mathrm{H})$ have high gene density.

The version 6 rice genome sequence coordinates, including chromosome, arm and base pair position, are included in Table S4 (Additional File 14), along with the chromosome and arm position from version 5. The 2943 genetically mapped barley SNPs were derived from 2786 source sequences, of which 2703 have a rice BLASTX match of at least $7 \times \mathrm{e}^{-5}$. A total of 36 of these had a best BLASTX against a gene positioned to different rice chromosomes when comparing ortholog locations in rice ver- 


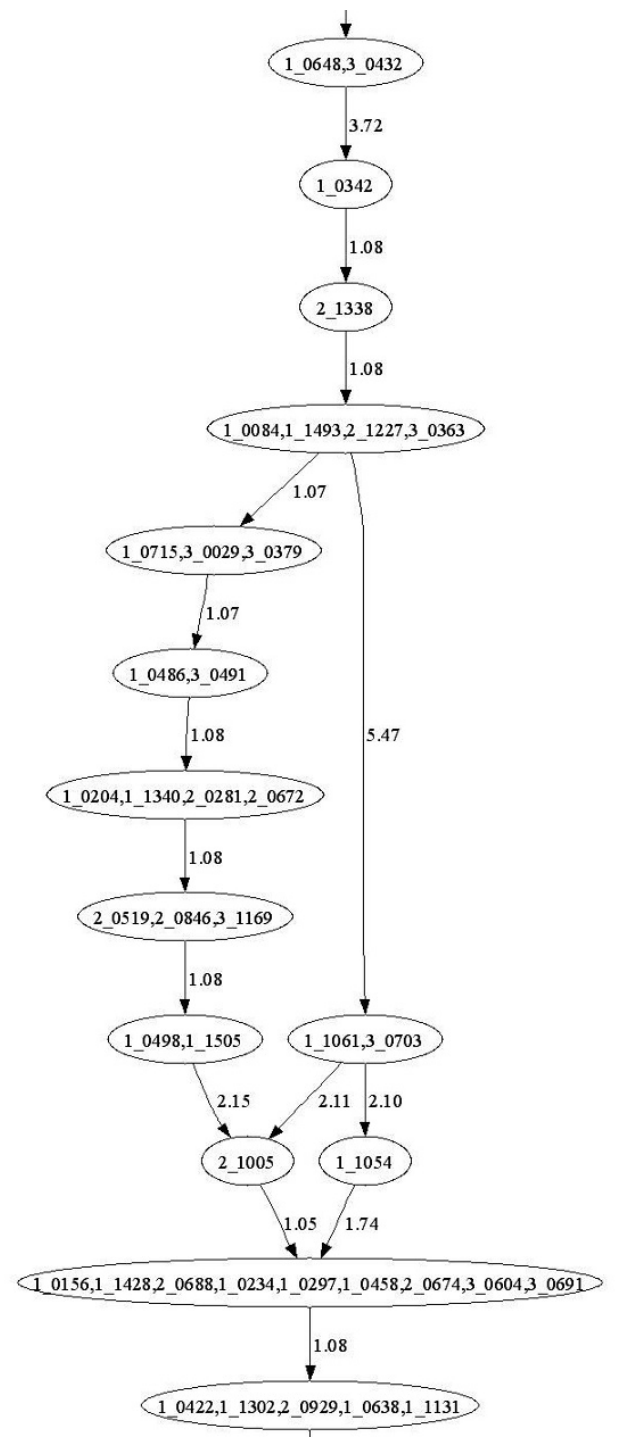

\section{Figure 4}

Segment of a consensus directed acyclic graph. A typical segment of a directed acyclic graph representing the consensus map of one barley linkage group is shown. Each oval represents one bin of SNP markers, using POPA names for SNPs. Where an oval contains more than one SNP, it means that there was no evidence of recombination in any mapping population between those markers. The observed recombination frequencies between marker bins are shown. The exact order of marker bins cannot be solved with certainty unless markers are shared between maps. Recombination frequencies are often not proportional to physical distance, nor consistent, when comparing two or more maps from different mapping populations. Therefore directed acyclic graphs provide a more exact description of the limit of knowledge of the marker order than does a linear map derived using approximations based on recombination values. See the text for further discussion. sion 5 to version 6 . It is interesting that 14 of the $36(39 \%)$ changes in the rice genome annotations brought rice-barley synteny into line with the barley genetic map, 9 of the $36(25 \%)$ changes degraded rice-barley synteny and 12 (33\%) had a neutral effect because neither the version 5 nor 6 rice annotations were syntenic with barley. One had a neutral effect because both BLAST hits were consistent with syntenic duplications in the barley and rice genomes. It is not unusual to find imperfect synteny such as the 12 of $36(33 \%)$ revised but non-syntenic positions; in fact 745 of the 2703 mapped barley SNP source sequences $(27.6 \%)$ do not point to a best rice BLASTX within the major synteny block. However, from this comparison of the edited positions in rice versions 5 versus 6 to the 2943 SNP barley genetic linkage map, it appears that the barley SNP map is the more stable point of reference. Consequently, it may be of some benefit to use the barley genetic map for further revisions of the rice genome sequence.

\section{BOPAI and BOPA2 elements and performance}

As discussed above, the two production OPAs, BOPA1 and BOPA2, had somewhat different design elements. These differences have been reflected in the performance of BOPA1 and BOPA2 for the genotyping of breeding germplasm within the BarleyCAP project [17]. Table 2 provides a comparison of BOPA1 and BOPA2 in relation to both SNP representation and the performance on 960 year 2006 US breeder DNA samples in the BarleyCAP project. Table 2 also summarizes information provided in greater detail in Table S4 (Additional File 14) on the relationship of BOPA SNPs to probe sets on the Affymetrix Barley1 GeneChip [18] and the 2943 mapped SNPs in the present work. Extensive analyses of the diversity of breeding germplasm will be the subject of other papers; here we emphasize only the elements and fundamental performance characteristics of BOPA1 and BOPA2. One can see that BOPA1, which was designed using only SNPs with a minor allele frequency (MAF) of at least 0.08 in the design germplasm, yielded MAF values less than 0.05 for only 164 SNPs $(10.7 \%)$ in the US breeding materials. In contrast BOPA2, which targeted 615 SNPs with MAF less than 0.08 in the design germplasm, yielded MAF values less than 0.05 for 585 SNPs (38.1\%) in the breeding germplasm. This included about three times as many SNPs with $\mathrm{MAF}=0$ (301 versus 99) and 4.4 times as many SNPs (284/65) with MAF between 0 and 0.05 . Thus, BOPA2 has greater sensitivity to detect rare alleles than does BOPA1, some of which may be important for the development of new varieties containing uncommon alleles of certain genes. But, this increased sensitivity is counterbalanced by a compromise in the reduced frequency of informative SNPs in general. 


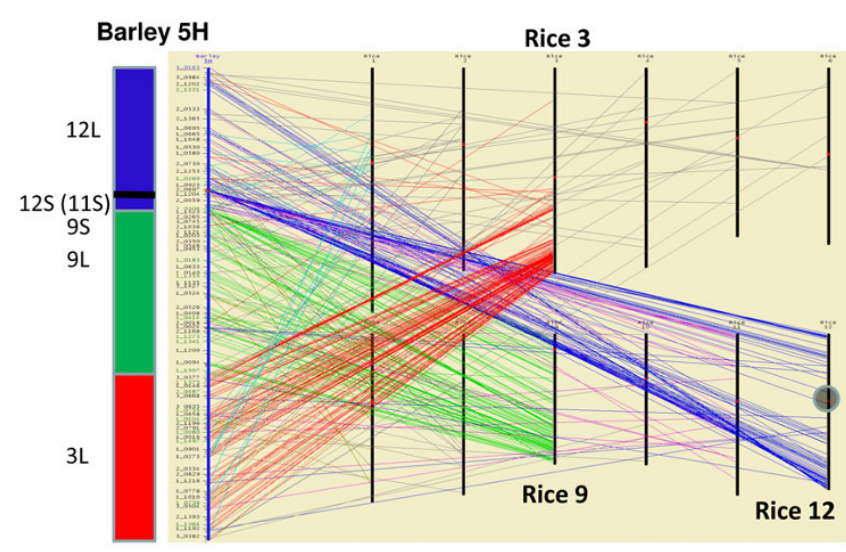

Figure 5

Barley-rice synteny in detail for $\mathbf{5 H}$. HarvEST screenshot showing barley-rice synteny for chromosome $5 \mathrm{H}$. Colored lines connect each barley locus to the position of the best BLAST hit on the rice genome.

Table S5 (Additional File 15) lists the MAF values determined during the design of BOPA1 and BOPA2 versus the observed MAF values in year 2006 and year 2007 BarleyCAP breeding germplasm. For example, 11 of 157 SNPs with a design MAF of 0.01 or lower had an observed MAF of at least 0.08 in year 2006 or 2007 breeding germplasm. Similarly, 25 of 283 SNPs with a design MAF of 0.024 or lower had an observed MAF of at least 0.10 in year 2006 or 2007 breeding germplasm. The differences between BOPA1 and BOPA2 should be carefully considered by potential users, and the characteristics of specific SNPs should be considered when selecting subsets of SNPs for other platforms.

\section{Other characteristics of the 2943 SNP map}

It is perhaps of relevance that there were significant differences in the genetic length of some of the individual chromosomes in the different populations (Table 1). For example, the genetic length of chromosome $4 \mathrm{H}$ in the SxM population is expanded relative to the same chromosome in any other population, and all of the other chromosomes have a longer genetic length in the OWB population than in the other populations. Also, the genetic map lengths are consistently higher than would be expected from cytogenetic counts of chiasmata per meiosis for this species, as previously noted [19] despite the fact that methodological errors in genotyping can be ruled out in the present work because of the lack of any suspicious-looking singleton double recombinants. The notable deviations from mean genetic distance values indicate that the genetic background as well as environmental factors may have had a significant effect on recombination, and presumably also chiasmata counts, in this species. Also, although the broad patterns of synteny within grasses recognized previously by many investigators has been confirmed by this work, the hitherto unprecedented density of gene-derived markers enable further delineation of several inversions and rearrangements of gene order at macro-, meso- and micro-synteny levels. Chromosome 5H (Figure 5) provides one example of such rearrangements at the macrosynteny level. The HarvEST:Barley http://harvest.ucr.edu synteny viewer provides zoom-in functionality to enable visualization at meso and micro-synteny levels as well.

\section{Access to the linkage map and SNP data}

The 2943 SNP linkage map can be accessed by several browsers including HarvEST:Barley [14] or [20], GrainGenes [21], NCBI [22] and THT [23]. New versions of the map may become available as additional mapping populations are applied to BOPA1 and BOPA2, linkage disequilibrium is used for mapping and the physical map and genome sequence are coupled to the genetic linkage map.

\section{Conclusion}

The unprecedented density of genic markers and marker bins enabled a high resolution comparison of the genomes of barley and rice. Low recombination in pericentric regions is evident from bins containing many more than the average number of markers, meaning that a large number of genes are recombinationally locked into the genetic centromeric regions of several barley chromosomes. Examination of US breeding germplasm illustrated the usefulness of BOPA1 and BOPA2 in that they provide excellent marker density and sensitivity for detection of minor alleles in this genetically narrow material.

\section{Methods \\ Five I536-SNP GoldenGate assays (Figure I, Table 2)}

Three pilot-phase 1536-SNP GoldenGate assays were developed. These "pilot OPAs" are referred to as POPA1, POPA2 and POPA3. Two 1536-SNP production-scale OPAs, referred to as BOPA1 and BOPA2, were developed from SNPs tested on the pilot OPAs. All sequences used as SNP sources were generated using the Sanger dideoxy chain termination method.

\section{POPAI and POPA2}

The contents of POPA1 and POPA2 came from an initial list of SNPs comprised of the union of three intersecting lists from SCRI (1,658 SNPs), IPK (985 SNPs) and UCR $(12,615$ SNPs). SCRI and IPK SNPs were derived from PCR amplicon sequences, whereas UCR SNPs were derived nearly entirely from EST sequences. In the selection of SNPs for the OPAs, preference was given to SNPs derived from amplicon sequences. Nearly all SNPs on POPA 1 and about $60 \%$ of the SNPs on POPA 2 targeted stress-regulated genes. The composition of POPA1 included 1524 barley SNPs, one per gene, of which 1033 


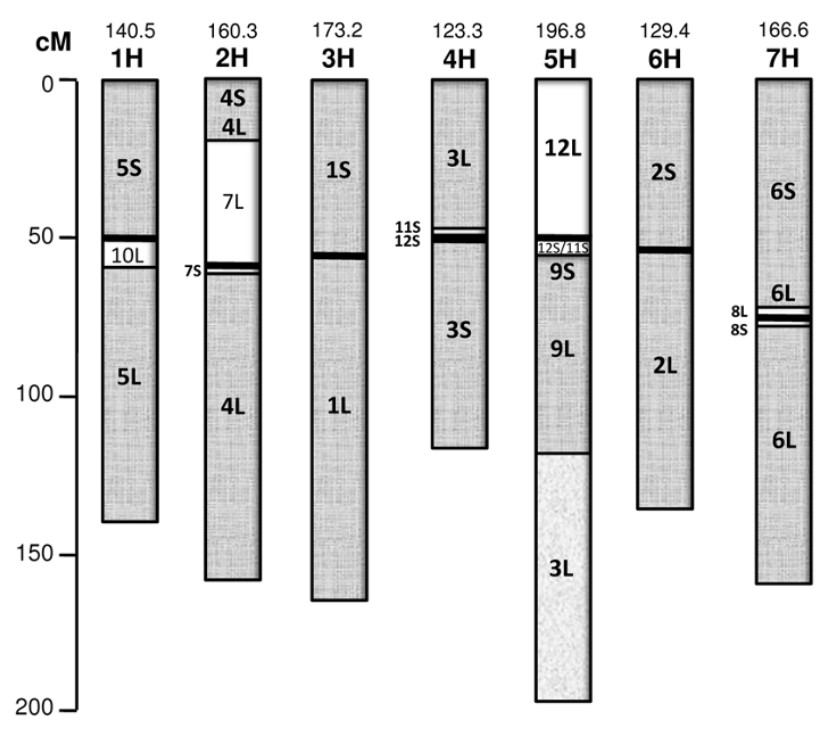

Figure 6

Barley-rice synteny summary. Seven barley linkage groups represented as rice synteny blocks. Numbers inside each barley chromosome indicate syntenic rice chromosome arm.

were derived from ESTs and 491 from amplicon sequences. The composition of POPA2 included 1536 barley SNPs, one per gene including 258 genes represented on POPA1, of which 1456 were from ESTs and 80 from amplicon sequences.

\section{BOPAI}

BOPA1 represented 705 SNPs from POPA1 and 832 from POPA2, including one SNP in common. All BOPA1 SNPs had a satisfactory technical performance on POPA1 or POPA2 and a minor allele frequency of at least 0.08 . To the extent of results presented in this manuscript, BOPA1 included 1414 mapped and 122 unmapped SNPs.

\section{POPA3}

Residual SNPs from the sources of POPA1 and POPA2 were insufficient to complete the design of POPA3 without compromising on the SNP selection criteria. Additional SNPs for POPA3 came from three sources: 1) an extended list of 5,732 SNPs identified in SCRI amplicon sequences, 2) colleagues who contributed SNPs from amplicon sequences of specific genes of biological interest and 3) an expanded barley EST resource. The first two of these additional sources were exhausted for POPA3 design. In the selection of EST-derived SNPs, priority was given to genes previously classified as having interesting expression patterns during malting or upon exposure to pathogens, or relevant to malting, brewing quality, abiotic stress or phenology. The composition of POPA3 included
1536 barley SNPs, in many cases more than one per gene and in some cases including genes represented on POPA1 or POPA2. In total, 967 POPA3 SNPs were derived from ESTs and 569 from amplicon sequences.

\section{BOPA2}

BOPA2 represented 406 SNPs from POPA1, 178 from POPA2 and 952 from POPA3. The primary emphases of BOPA2 were representation of mapped SNPs that were not included on BOPA1 and inclusion of multiple SNPs for certain genes to reveal haplotypes at these loci, with some weight given to MAF. BOPA2 contained 921 SNPs with MAF at least $0.08,256$ SNPs with MAF at least 0.04 but less than $0.08,345$ SNPs with MAF least 0.005 but less than 0.04 , and 14 SNPs with only one allele $(\mathrm{MAF}=0)$ in the germplasm examined using POPA3. To the extent of results presented in this manuscript, BOPA2 included 1263 mapped and 273 unmapped SNPs. A total of 967 SNPs were from ESTs and 569 from amplicon sequences.

\section{SNP annotations}

Table S4 (Additional File 14) provides alternative SNP names arising from this work, and several annotation fields for all SNPs represented on POPA1, POPA2, POPA3, BOPA1 and BOPA2. The annotations include BLAST hits to the rice and Arabidopsis genomes and UniProt, the relationship of SNP source sequences to HarvEST:Barley unigenes and probe sets on the Affymetrix Barley1 GeneChip and source consensus sequences. To assign SNP loci on the genetic map to chromosome arms, centromere positions were identified using flow-sorted chromosome arms following the method described in Simkova et al. [24]; results of this work will be described elsewhere (Bhat et al., in preparation). The annotation information in Table S4 (Additional File 14) is also available from HarvEST:Barley [14] and [20]. The HarvEST BLAST server [25] provides the 2943 mapped SNP unigene sequences as a searchable database.

\section{DNA sources}

Genomic DNAs of 93 doubled haploid maplines and the parents (Dom, Rec) of the Oregon Wolfe Barley (OWB) population [26,27] 148 doubled haploids and the parents of the Steptoe $\times$ Morex $(\mathrm{SxM})$ population [7,28], 95 doubled haploid maplines and the parents of the Haruna Nijo $\times$ OHU602 (HxO) population and 213 additional germplasm samples were purified using Plant DNeasy (Qiagen, Valencia, CA, USA) starting with $100-300 \mathrm{mg}$ of young seedling leaves. Genomic DNAs of 93 doubled haploid maplines and the Barke parent from the Morex $\times$ Barke population (Stein et al. unpublished) were produced using a CTAB method. All DNA samples were checked for concentration using UV spectroscopy and Quant-iT PicoGreen (Invitrogen, Carlsbad, CA, USA) and adjusted to approximately $120 \mathrm{ng} / \mu \mathrm{l}$ in TE buffer. 
Table 2: Design and performance characteristics of BOPAI and BOPA2

\begin{tabular}{|c|c|c|c|}
\hline & BOPAI & BOPA2 & Both \\
\hline SNPs represented & 1536 & 1536 & 3072 \\
\hline Number of unigenes on other BOPA* & 77 & 77 & NA \\
\hline Unigenes represented & 1536 & 1442 & 2901 \\
\hline Number of unigenes with I SNP & 1536 & 1380 & 2770 \\
\hline Number of unigenes with 2 SNPs & 0 & 43 & 106 \\
\hline Number of unigenes with 3 SNPs & 0 & 11 & 16 \\
\hline Number of unigenes with 4 SNPs & 0 & 3 & 3 \\
\hline Number of unigenes with 5 SNPs & 0 & 5 & 6 \\
\hline SNPs included in 2943 mapped & 1414 & 1263 & 2677 \\
\hline SNP unigenes matching Barley I probe set(s) & 1489 & 1433 & 2921 \\
\hline MAF $\geq 0.08$ in design germplasm & 1536 & 921 & 2457 \\
\hline MAF $\geq 0.04$ and $<0.08$ in design germplasm & 0 & 256 & 256 \\
\hline MAF $\geq 0.005$ and $<0.04$ in design germplasm & 0 & 345 & 345 \\
\hline MAF = 0 in design germplasm & 0 & 14 & 14 \\
\hline MAF = 0 in 2006 BarleyCAP genotypes & 99 & 301 & 400 \\
\hline MAF $>0$ and $<0.05$ in 2006 BarleyCAP genotypes & 65 & 284 & 349 \\
\hline MAF $\geq 0.05$ in 2006 BarleyCAP genotypes & 1372 & 951 & 2323 \\
\hline
\end{tabular}

*Among the 77 unigenes represented by SNPs on both BOPAs, 69 have I SNP on BOPA2, 6 have 2 SNPs on BOPA2, I has three SNPs on BOPA2, I has four SNPs on BOPA2.

\section{Data production for map construction and MAF estimation}

DNA concentrations were re-checked using Quant-iT PicoGreen (Invitrogen, Carlsbad, CA) and standardized to $80 \mathrm{ng} / \mu \mathrm{l}$ in TE buffer in preparation for the GoldenGate assay and $5 \mu \mathrm{l}$ (400 ng) were used for each assay. Data were generated from each progeny line in the OWB, SxM and MxB doubled haploid populations using POPA1 and POPA2. Data were also produced using POPA3 from the complete OWB and MxB sets of DNA samples, but from only 92 SxM doubled haploids. Data from $95 \mathrm{HxO}$ doubled haploids using BOPA1 were also generated. For each of these four mapping populations, extensive integration of SNP data with other types of marker data will be described elsewhere (for example OWB marker integration in Szúcs et al. [10]). Data used for the determination of allele frequency (see below) came from 125 germplasm samples for POPA1, 195 germplasm samples for POPA2, and 189 germplasm samples for POPA3.

\section{Data processing}

Raw data were transformed to genotype calls, initially using Illumina GenCall and subsequently using Illumina BeadStudio version 3 with the genotyping module. For each OPA, the data from all samples were visually inspected to manually set 1536 archetypal clustering patterns. The cluster positioning was guided by knowledge that heterozygotes are nearly non-existent in doubled haploids and rare in highly inbred parental genotypes and germplasm samples. Several "synthetic heterozygote" DNA samples were made by mixing parental DNAs in a 1:1 mass ratio (Figure 2A, green dots), and included to anchor heterozygote cluster positions to enable the identification of true heterozygotes which occur at a significant 
frequency in germplasm samples that have not been sufficiently inbred to reach a state of genome-wide allele fixation. The spatial positions of heterozygote and homozygote data clusters were confined to areas of high certainty so that data points with less certainty fell outside the boundaries of heterozygotes and homozygotes and were scored as "no-call" (Fig 2A, one germplasm sample as black dot). Polymorphisms with theta compressed clusters were not used if the compression was such that any homozygote call was not plainly distinguishable (Figure 2B, set as Gentrain 0.000, 100\% "no call"). Vertically separated data clusters were not accepted as polymorphisms (Figure 2C). Following the production of one master workspace for each Pilot OPA using all DNA samples, customized workspaces were produced for each mapping population to optimize the genotype calls via minor adjustments of cluster positions. Genotype calls were exported as spreadsheets from BeadStudio and then parsed to create input for mapping programs.

\section{Individual and consensus map production}

Individual maps were made principally using MSTMap $[29,30]$ for each data set from the four doubled haploid mapping populations. In brief, MSTMap first identifies linkage groups, then determines marker order by finding the minimum spanning tree of a graph for each linkage group, then calculates distances between marker using recombination frequencies. JoinMap 4 [31] was used to confirm linkage groups and marker order determined by MSTMap. Raw data for problematic markers were reviewed using BeadStudio and then either the marker was discarded entirely if any ambiguity in data calling could not be resolved or individual genotype calls were modified if it was plainly evident that such adjustments were warranted. Each such review of primary data was followed by the production of new maps; this iterative process generally involved 10-20 cycles for each individual map. At several points, a consensus map was produced using MergeMap [32], which also flags problematic markers for review. MergeMap takes into account marker order from individual maps and calculates a consensus marker order. Briefly, the input to MergeMap is a set of directed acyclic graphs (DAGs) [33] from each individual map, and the output is a set of consensus DAGs (Figure 3, Figures S3-S9, Additional Files 6, 7, 8, 9, 10, 11, 12), where each is consistent with all (or nearly all) of the markers in the individual input maps. MergeMap then linearizes each consensus DAG using a mean distance approximation. The consensus map coordinates from MergeMap were normalized to the arithmetic mean $\mathrm{cM}$ distance for each linkage group from the four individual maps (Figure S2, see Additional File 4 and Table S4, see Additional File 14).

\section{Implementation of BOPAI and BOPA2 in US barley breeding germplasm}

As part of Barley CAP [17], the two BOPAs have been part of an effort to genotype a total of 3840 US barley breeding lines contributed from ten US barley breeding programs for association mapping analyses. As of January 2009, data from both BOPAs had been generated for 1920 breeding lines, with 960 submitted from the selections of each of two years, 2006 and 2007. Table S5 (Additional File 15) provides MAF for observed in these samples for each SNP in BOPA1 and BOPA2.

\section{Abbreviations}

AFLP: amplified fragment length polymorphism; DAG: directed acyclic graph; DArT: diversity array technology; EST: expressed sequence tag; QTL: quantitative trait locus; RFLP: restriction fragment length polymorphism; SNP: single nucleotide polymorphism; SSR: simple sequence repeat; STS: sequence tagged site.

\section{Authors' contributions}

The contributions of authors and other colleagues, locations of work conducted and cost sharing are detailed in Supplemental Text (Additional File 1), which contains citations of references 34-39 and Additional Files 16, 17, $18,19,20$ and is essentially an expanded version of Methods.

\section{Additional material}

Additional file 1
Supplemental Text
Click here for file
[http://www.biomedcentral.com/content/supplementary/1471-
2164-10-582-S1.DOC]

\section{Additional file 2}

Figure S1. SNP yield. The near-linear relationship between the number of SNPs and the product of the number of EST sequences for pairwise genotype comparisons is shown by plotting all values versus a linear regression line. Each axis is on a logarithmic scale. Oval shapes indicate a comparison involving the wild barley accession OHU602. See text for additional details.

Click here for file

[http://www.biomedcentral.com/content/supplementary/14712164-10-582-S2.DOC]

\section{Additional file 3}

Table S1. All individual and consensus maps, including SxM bin markers.

Click here for file

[http://www.biomedcentral.com/content/supplementary/14712164-10-582-S3.XLS] 


\section{Additional file 4}

Figure S2. Consensus 2943 SNP genetic linkage map.

Click here for file

[http://www.biomedcentral.com/content/supplementary/1471-

2164-10-582-S4.DOC]

\section{Additional file 5}

Table S2. All data from $M x B, O W B$ and SxM mapping populations. Click here for file

[http://www.biomedcentral.com/content/supplementary/14712164-10-582-S5.XLS]

\section{Additional file 6}

Figure S3. Complete consensus directed acyclic graphs for barley chromosomes $1 \mathrm{H}$.

Click here for file

[http://www.biomedcentral.com/content/supplementary/1471-

2164-10-582-S6.JPEG]

\section{Additional file 7}

Figure S4. Complete consensus directed acyclic graphs for barley chromosomes $2 \mathrm{H}$.

Click here for file

[http://www.biomedcentral.com/content/supplementary/1471-

2164-10-582-S7.JPEG]

\section{Additional file 8}

Figure S5. Complete consensus directed acyclic graphs for barley chromosomes $3 H$.

Click here for file

[http://www.biomedcentral.com/content/supplementary/14712164-10-582-S8.JPEG]

\section{Additional file 9}

Figure S6. Complete consensus directed acyclic graphs for barley chromosomes $4 \mathrm{H}$.

Click here for file

[http://www.biomedcentral.com/content/supplementary/1471-

2164-10-582-S9.JPEG]

\section{Additional file 10}

Figure S7. Complete consensus directed acyclic graphs for barley chromosomes $5 \mathrm{H}$.

Click here for file

[http://www.biomedcentral.com/content/supplementary/1471-

2164-10-582-S10.JPEG]

\section{Additional file 11}

Figure S8. Complete consensus directed acyclic graphs for barley chromosomes $6 \mathrm{H}$.

Click here for file

[http://www.biomedcentral.com/content/supplementary/14712164-10-582-S11.JPEG]

\section{Additional file 12}

Figure S9. Complete consensus directed acyclic graphs for barley chromosomes $7 \mathrm{H}$.

Click here for file

[http://www.biomedcentral.com/content/supplementary/14712164-10-582-S12.JPEG]

\section{Additional file 13}

Table S3. Synonymous marker names.

Click here for file

[http://www.biomedcentral.com/content/supplementary/14712164-10-582-S13.DOC]

\section{Additional file 14}

Table S4. All marker consensus map coordinates, names, source types, BLASTs, probe sets, sequences.

Click here for file

[http://www.biomedcentral.com/content/supplementary/14712164-10-582-S14.XLS]

\section{Additional file 15}

Table S5. Minor allele frequencies for each SNP on BOPA1 and BOPA2. Click here for file

[http://www.biomedcentral.com/content/supplementary/14712164-10-582-S15.XLS]

\section{Additional file 16}

Table S6. POPA1 SNPS.

Click here for file

[http://www.biomedcentral.com/content/supplementary/14712164-10-582-S16.XLS]

\section{Additional file 17}

Table S7. POPA2 SNPS.

Click here for file

[http://www.biomedcentral.com/content/supplementary/14712164-10-582-S17.XLS]

\section{Additional file 18}

Table S8. POPA3 SNPS.

Click here for file

[http://www.biomedcentral.com/content/supplementary/14712164-10-582-S18.XLS]

\section{Additional file 19}

\section{Table S9. BOPA1 SNPs.}

Click here for file

[http://www.biomedcentral.com/content/supplementary/14712164-10-582-S19.XLS]

\section{Additional file 20}

\section{Table S10. BOPA2 SNPs.}

Click here for file

[http://www.biomedcentral.com/content/supplementary/14712164-10-582-S20.XLS]

\section{Acknowledgements}

This research was supported by NSF DBI Grant No. 0321756 "Coupling EST and Bacterial Artificial Chromosome Resources to Access the Barley Genome", USDA-CSREES-NRI Grant No. 2006-55606-16722 "Barley Coordinated Agricultural Project: Leveraging Genomics, Genetics, and Breeding for Gene Discovery and Barley Improvement", the Biotechnology and Biological Sciences Research Council and Scottish Executive Environment and Rural Affairs Department, and core funding from the Leibniz Institute of Plant Genetics and Crop Plant Research. 


\section{References}

I. Rostoks N, Mudie S, Cardle L, Russell J, Ramsay L, Booth A, Svensson JT, Wanamaker SI, Walia H, Rodriguez EM, Hedley PE, Liu H, Morris J, Close TJ, Marshall DF, Robbie Waugh R: Genome-wide SNP discovery and linkage analysis in barley based on genes responsive to abiotic stress. Molecular Genetics and Genomics 2005 , 274:5।5-527.

2. Wenzl P, Li H, Carling J, Zhou M, Raman H, Paul E, Hearnden P, Maier C, Xia L, Caig V, Ovesná J, Cakir M, Poulsen D, Wang J, Raman R, Smith KP, Muehlbauer GJ, Chalmers KJ, Kleinhofs A, Huttner E, Kilian A: A high-density consensus map of barley linking DArT markers to SSR, RFLP and STS loci and agricultural traits. BioMed Central Genomics 2006, 7:206.

3. Marcel TC, Varshney RK, Barbieri M, Jafary H, de Kock MJD, Graner A, Niks RE: high-density consensus map of barley to compare the distribution of QTLs for partial resistance of Puccinia hordei $\mathbf{A}$ and of defence gene homologues. Theoretical and Applied Genetics 2007, I | 4:487-500.

4. Stein N, Prasad M, Scholz U, Thiel T, Zhang H, Wolf M, Kota R, Varshney RK, Perovic D, Grosse I, Graner A: A I,000-loci transcript map of the barley genome: new anchoring points for integrative grass genomics. Theoretical and Applied Genetics 2007, I | 4:823-839.

5. Varshney RK, Marcel TC, Ramsay L, Russell J, Röder MS, Stein N Waugh R, Langridge P, Niks RE, Graner A: A high density barley microsatellite consensus map with 775 SSR loci. Theoretical and Applied Genetics 2007, I | 4:109|- I 103.

6. Potokina E, Druka A, Luo Z, Wise R, Waugh R, Kearsey M: Gene expression quantitative trait locus analysis of 16,000 barley genes reveals a complex pattern of genome-wide transcriptional regulation. Plant Journal 2008, 53:90-I0I.

7. Kleinhofs A, Kilian A, Saghai Maroof MA, Biyashev RM, Hayes P, Chen FQ, Lapitan N, Fenwick A, Blake TK, Kanazin V, Ananiev E, Dahleen L, Kudrna D, Bollinger J, Knapp SJ, Liu B, Sorrells M, Heun M, Franckowiak JD, Hoffman D, Skadsen R, Steffenson B]: A molecular, isozyme and morpohological map of the barley (Hordeum vulgare) genome. Theoretical and Applied Genetics 1993, 86:705-7I2.

8. Hearnden PR, Eckermann PJ, McMichael GL, Hayden MJ, Eglinton JK, Chalmers KJ: A genetic map of I,000 SSR and DArT markers in a wide barley cross. Theoretical and Applied Genetics 2007, I | 5:383-39|.

9. Sato K, Nankaku N, Takeda K: A high density transcript linkage map of barley derived from a single population. Heredity 2009 , 103: I10-117.

10. Szücs P, Blake VC, Bhat PR, Close TJ, Cuesta-Marcos A, Muehlbauer G], Ramsay LV, Waugh R, Hayes PM: An integrated resource for barley linkage map and malting quality QTL alignment. The Plant Genome 2009, 2: 134-140.

II. Kleinhofs A, Graner A: An integrated map of the barley genome. In DNA Markers in Plants Edited by: Vasil IK. Dordrecht, The Netherlands: Kluwer Academic; 2001:187-199.

12. Kota R, Varshney RK, Prasad M, Zhang H, Stein N, Graner A: ESTderived single nucleotide polymorphism markers for assembling genetic and physical maps of the barley genome. Functional and Integrative Genomics 2008, 8:223-233.

13. Rice Genome Annotation Project Database [http://rice.plant biology.msu.edu/pseudomolecules/info.shtml]

14. HarvEST:Barley [http://harvest.ucr.edu]

15. Sorrells ME, LaRota M, Bermudez-Kandianis CE, Greene RA, Kantety R, Munkvold JD, Miftahudin, Mahmoud A, Ma X, Gustafson PJ, Qi LL, Echalier B, Gill BS, Matthews DE, Lazo GR, Chao S, Anderson OD, Edwards H, Linkiewicz AM, Dubcovsky J, Akhunov ED, Dvorak J, Zhang D, Nguyen HT, Peng J, Lapitan NL, Gonzalez-Hernandez JL, Anderson JA, Hossain K, Kalavacharla V, Kianian SF, Choi DW, Close T], Dilbirligi M, Gill KS, Steber C, Walker-Simmons MK, McGuire PE, Qualset CO: Comparative DNA sequence analysis of wheat and rice genomes. Genome Research 2003, I3:1818-1827.

16. Salse J, Bolot S, Throuds M, Jouffe V, Piegu B, Quraishi UM, Calcagno $\mathrm{T}$, Cooke R, Delseny $M$, Feuillet $C$ : Identification and characterization of shared duplications between rice and wheat provide new insight into grass genome evolution. Plant Cell 2008, 20:II-24.

17. The barley Coordinated Agricultural Project (CAP) [http:// www.barleycap.org]

18. Close TJ, Wanamaker SI, Caldo RA, Turner SM, Ashlock DA, Dickerson JA, Wing RA, Muehlbauer GJ, Kleinhofs A, Wise RP: A new resource for cereal genomics: 22K barley GeneChip comes of age. Plant Physiology 2004, I 34:960-968.

19. Nilsson NO, Sall T, Bengston BO: Chiasma and recombination data in plants - are they compatible? Trends in Genetics 1993, 9:344-348.

20. HarvEST:Web [http://www.harvest-web.org]

21. GrainGenes [http://wheat.pw.usda.gov/GG2/index.shtml]

22. National Center for Biotechnology Information [http:// www.ncbi.nlm.nih.gov/]

23. The Hordeum Toolbox [http://www.hordeumtoolbox.org/]

24. Šimková $H$, Svensson JT, Condamine $P, H$ řibová E, Suchánková $P$, Bhat PR, Bartoš J, Šafář J, Close TJ, Doležel J: Coupling amplified DNA from flow-sorted chromosomes to high-density SNP mapping in barley. BMC Genomics 2008, 9:294

25. The HarvEST BLAST Server [http://138.23.191.145/blast/ index.html]

26. Costa JM, Corey A, Hayes PM, Jobet C, Kleinhofs A, Kopsich-Obusch A, Kramer SF, Kudrna D, Li M, Riera-Lizarazu O, Sato K, Szűcs P, Toojinda T, Vales MI, Wolfe RI: Molecular mapping of the Oregon Wolfe Barleys: a phenotypically polymorphic doubledhaploid population. Theoretical and Applied Genetics 2001, 103:4I5-424.

27. Barley World [http://barleyworld.org/oregonwolfe.php]

28. GrainGenes:The Steptoe × Morex Barley Mapping Population [http://wheat.pw.usda.gov/ggpages/SxM/]

29. Wu Y, Bhat PR, Close TJ, Lonardi S: Efficient and accurate construction of genetic linkage maps from the minimum spanning tree of a graph. PLoS Genetics 2008, 4(10):el0002I 2.

30. MSTmap Online [http:// 38.23.191.145/mstmap/]

31. Kyazma JoinMap [http://www.kyazma.nl/index.php/mc.JoinMap/]

32. Wu Y, Close TJ, Lonardi S: On the accurate construction of consensus genetic maps. Proceedings of LSS Computational Systems Bioinformatics Conference: 26-29 August2008; Stanford 2008:285-296.

33. Yap IV, Schneider D, Kleinberg J, Matthews D, Cartinhour S, McCouch SR: A graph-theoretic approach to comparing and integrating genetic, physical and sequence-based maps. Genetics 2003, I 65:2235-2247.

34. Walia H, Wilson C, Wahid A, Condamine P, Cui X, Close T]: Expression analysis of barley (Hordeum vulgare L.) during salinity stress. Functional and Integrative Genomics 2006, 6: I43-I56.

35. Svensson JT, Crosatti C, Campoli C, Bassi R, Stanca AM, Close TJ, Cattivelli L: Transcriptome analysis of cold acclimation in barley Albina and Xantha mutants. Plant Physiology 2006, I 4 I:257-270.

36. Tommasini L, Svensson JT, Rodriguez EM, Wahid A, Malatrasi M, Kato K, Wanamaker S, Resnik J, Close TJ: Dehydrin gene expression provides an indicator of low temperature and drought stress: transcriptome-based analysis of barley (Hordeum vulgare L.). Functional and Integrative Genomics 2008, 8:387-405.

37. Huang XM, Madan A: CAP3: a DNA sequence assembly program. Genome Research 1999, 9:868-877.

38. Cui X, Xu J, Asghar R, Condamine P, Svensson JT, Wanamaker S, Stein N, Roose $M$, Close TJ: Detecting single-feature polymorphisms using oligonucleotide arrays and robustified projection pursuit. Bioinformatics 2005, 21 :3852-3858.

39. Maplnspect [http://www.plantbreeding.wur.nl/UK/ software mapinspect.html]

Publish with Biomed Central and every scientist can read your work free of charge

"BioMed Central will be the most significant development for disseminating the results of biomedical research in our lifetime. "

Sir Paul Nurse, Cancer Research UK

Your research papers will be:

- available free of charge to the entire biomedical community

- peer reviewed and published immediately upon acceptance

- cited in PubMed and archived on PubMed Central

- yours - you keep the copyright
BioMedcentral 